\title{
Pacific
}

Journal of

Mathematics

\section{BIHARMONIC HYPERSURFACES IN RIEMANNIAN MANIFOLDS}

\author{
YE-LIN OU
}




\title{
BIHARMONIC HYPERSURFACES IN RIEMANNIAN MANIFOLDS
}

\author{
YE-LIN OU
}

\begin{abstract}
We study biharmonic hypersurfaces in a generic Riemannian manifold. We first derive an invariant equation for such hypersurfaces generalizing the biharmonic hypersurface equation in space forms studied by Jiang, Chen, Caddeo, Montaldo, and Oniciuc. We then apply the equation to show that the generalized Chen conjecture is true for totally umbilical biharmonic hypersurfaces in an Einstein space, and construct a 2-parameter family of conformally flat metrics and a 4-parameter family of multiply warped product metrics, each of which turns the foliation of an upper-half space of $\mathbb{R}^{m}$ by parallel hyperplanes into a foliation with each leaf a proper biharmonic hypersurface. We also study the biharmonicity of Hopf cylinders of a Riemannian submersion.
\end{abstract}

\section{Biharmonic maps and submanifolds}

All manifolds, maps, and tensor fields that appear in this paper are assumed to be smooth unless stated otherwise.

A biharmonic map is a map $\varphi:(M, g) \rightarrow(N, h)$ between Riemannian manifolds that is a critical point of the bienergy functional

$$
E^{2}(\varphi, \Omega)=\frac{1}{2} \int_{\Omega}|\tau(\varphi)|^{2} \mathrm{~d} x
$$

for every compact subset $\Omega$ of $M$, where $\tau(\varphi)=$ Trace $_{g} \nabla \mathrm{d} \varphi$ is the tension field of $\varphi$. The Euler-Lagrange equation of this functional gives the biharmonic map equation [Jiang 1986b]

$$
\tau^{2}(\varphi):=\operatorname{Trace}_{g}\left(\nabla^{\varphi} \nabla^{\varphi}-\nabla_{\nabla^{M}}^{\varphi}\right) \tau(\varphi)-\operatorname{Trace}_{g} R^{N}(\mathrm{~d} \varphi, \tau(\varphi)) \mathrm{d} \varphi=0,
$$

which states that $\varphi$ is biharmonic if and only if its bitension field $\tau^{2}(\varphi)$ vanishes identically. In this equation we used $R^{N}$ to denote the curvature operator of $(N, h)$

MSC2000: 53C12, 58E20, 53C42.

Keywords: biharmonic maps, biharmonic hypersurfaces, biharmonic foliations, conformally flat space, Einstein space.

Supported by the Texas A\&M University-Commerce Faculty Development Program, 2008-2009. 
defined by

$$
R^{N}(X, Y) Z=\left[\nabla_{X}^{N}, \nabla_{Y}^{N}\right] Z-\nabla_{[X, Y]}^{N} Z .
$$

Clearly, it follows from (1) that any harmonic map is biharmonic and we call the nonharmonic biharmonic maps proper biharmonic maps.

Let $M^{m}$ be a submanifold of Euclidean space $\mathbb{R}^{n}$ with the mean curvature vector $H$ viewed as a map $H: M \rightarrow \mathbb{R}^{n}$. B. Y. Chen [1991] called $M^{m}$ a biharmonic submanifold if $\Delta H=\left(\Delta H^{1}, \ldots, \Delta H^{n}\right)=0$, where $\Delta$ is the Beltrami-Laplace operator of the induced metric on $M^{m}$. If we use $i: M \rightarrow \mathbb{R}^{n}$ to denote the inclusion map of the submanifold, then the tension field of the inclusion map $i$ is given by $\tau(i)=\Delta i=m H$, and hence the submanifold $M^{n} \subset \mathbb{R}^{n}$ is biharmonic if and only if $\triangle H=\triangle\left(\frac{1}{m} \triangle i\right)=\frac{1}{m} \triangle^{2} i=\frac{1}{m} \tau^{2}(i)=0$, that is, the inclusion map is a biharmonic map. In general, a submanifold $M$ of $(N, h)$ is called a biharmonic submanifold if the inclusion map $i:\left(M, i^{*} h\right) \rightarrow(N, h)$ is a biharmonic isometric immersion. It is well known that an isometric immersion is minimal if and only if it is harmonic. So a minimal submanifold is trivially biharmonic, and we call a nonminimal biharmonic submanifold a proper biharmonic submanifold.

Here are some known facts about biharmonic submanifolds:

Biharmonic submanifolds in Euclidean spaces. Jiang [1987] and then Chen and Ishikawa [1998] proved that any biharmonic submanifold in $\mathbb{R}^{3}$ is minimal. In [1992], Dimitrić showed that any biharmonic curve in $\mathbb{R}^{n}$ is a part of a straight line, any biharmonic submanifold of finite type in $\mathbb{R}^{n}$ is minimal, any pseudoumbilical submanifolds $M^{m} \subset \mathbb{R}^{n}$ with $m \neq 4$ is minimal, and any biharmonic hypersurface in $\mathbb{R}^{n}$ with at most two distinct principal curvatures is minimal. Hasanis and Vlachos [1995] proved that any biharmonic hypersurface in $\mathbb{R}^{4}$ is minimal. Based on these results, B. Y. Chen [1991] made the still-open conjecture that any biharmonic submanifold of Euclidean space is minimal.

Biharmonic submanifolds in hyperbolic space forms. Caddeo, Montaldo and Oniciuc [2002] showed that any biharmonic submanifold in hyperbolic 3-space is minimal, and that any $m$-dimensional pseudoumbilical biharmonic submanifold of hyperbolic $n$-space is minimal if $m \neq 4$. It is shown in [Balmuş et al. 2008] that any biharmonic hypersurface of hyperbolic $n$-space with at most two distinct principal curvatures is minimal. Based on these, Caddeo, Montaldo and Oniciuc [2001] extended Chen's conjecture to the generalized Chen conjecture: any biharmonic submanifold in $(N, h)$ is minimal if $\operatorname{Riem}^{N} \leq 0$.

Biharmonic submanifolds in spheres. The first example of a proper biharmonic submanifold in $S^{n+1}$ was found in [Jiang 1986a] to be the generalized Clifford torus $S^{p}(1 / \sqrt{2}) \times S^{q}(1 / \sqrt{2})$ with $p \neq q$ and $p+q=n$. Caddeo, Montaldo, and Oniciuc [2001] found a second type of proper biharmonic submanifolds in $S^{n+1}$ 
to be the hypersphere $S^{n}(1 / \sqrt{2})$, and also gave a complete classification of biharmonic submanifolds in $S^{3}$. Balmuş, Montaldo, and Oniciuc [2008] proved that any pseudoumbilical biharmonic submanifold $M^{m} \subset S^{n+1}$ with $m \neq 4$ has constant mean curvature, and also showed that if a hypersurface $M^{n} \subset S^{n+1}$ with at most two distinct principal curvatures (which by [Nishikawa and Maeda 1974] is equivalent for $n>3$ to saying that $M$ is a quasiumbilical or conformally flat hypersurface in $\left.S^{n+1}\right)$ is biharmonic, then $M$ is an open part of the hypersphere $S^{n}(1 / \sqrt{2})$ or the generalized Clifford torus $S^{p}(1 / \sqrt{2}) \times S^{q}(1 / \sqrt{2})$ with $p \neq q$ and $p+q=n$. Zhang [2008] found some examples of proper biharmonic real hypersurfaces in $\mathbb{C} P^{n}$ and determined all proper biharmonic tori $T^{n+1}=S^{1}\left(r_{1}\right) \times S^{1}\left(r_{2}\right) \times \cdots \times S^{1}\left(r_{n+1}\right)$ in $S^{2 n+1}$. All known examples of biharmonic submanifolds in spheres are consistent with the conjecture of [Balmuş et al. 2008] that any biharmonic submanifold in sphere has constant mean curvature, and any proper biharmonic hypersurface in $S^{n+1}$ is an open part of the hypersphere $S^{n}(1 / \sqrt{2})$ or the generalized Clifford torus $S^{p}(1 / \sqrt{2}) \times S^{q}(1 / \sqrt{2})$ with $p \neq q$ and $p+q=n$.

Biharmonic submanifolds in other model spaces. The survey article [Montaldo and Oniciuc 2006] contains an account of the study of biharmonic curves in various models. See [Arslan et al. 2005; Inoguchi 2004; Fetcu and Oniciuc 2009a; 2009b; Sasahara 2005; 2008] for special biharmonic submanifolds in contact manifolds or Sasakian space forms.

Biharmonic submanifolds in other senses. Some authors, for example, Javaloyes and Meroño [2003], use the condition $\Delta H=0$ to define a biharmonic submanifold of a Riemannian manifold; this definition agrees with ours only if the ambient space is flat. For conformal biharmonic submanifolds (that is, conformal biharmonic immersions), see [Ou 2009].

This paper studies biharmonic hypersurfaces in a generic Riemannian manifold. In Section 2, we derive an invariant equation for such hypersurfaces that involves the mean curvature function, the norm of the second fundamental form, the shape operator of the hypersurface, and the Ricci curvature of the ambient space. We prove that the generalized Chen conjecture holds for totally umbilical hypersurfaces in an Einstein space. Section 3 is devoted to constructing a family of conformally flat metrics and a family of multiply warped product metrics, each of which turns the foliation of an upper-half space of $\mathbb{R}^{m}$ by parallel hyperplanes into a foliation with each leaf a proper biharmonic hypersurface. We accomplish these by starting with hyperplanes in Euclidean space and then looking for a type of conformally flat or multiply warped product metric on the ambient space that will reduce the biharmonic hypersurface equation into ordinary differential equations whose solutions give the metrics that render the inclusion maps proper biharmonic isometric immersions. In Section 4, we study biharmonicity of Hopf cylinders 
given by a Riemannian submersion from a complete 3-manifold. Our method shows that there is no proper biharmonic Hopf cylinder in $S^{3}$, thus recovering [Inoguchi 2004, Proposition 3.1].

\section{The equations of biharmonic hypersurfaces}

Recall that if $\varphi: M \rightarrow(N, h)$ is the inclusion map of a submanifold, or more generally, an isometric immersion, then we have an orthogonal decomposition of the vector bundle $\varphi^{-1} T N=\tau M \oplus v M$ into the tangent and normal bundles. We use d $\varphi$ to identify $T M$ with its image $\tau M$ in $\varphi^{-1} T N$. Then, for any $X, Y \in \Gamma(T M)$ we have $\nabla_{X}^{\varphi}(\mathrm{d} \varphi(Y))=\nabla_{X}^{N} Y$, whereas $\mathrm{d} \varphi\left(\nabla_{X}^{M} Y\right)$ equals the tangential component of $\nabla_{X}^{N} Y$. It follows that

$$
\nabla \mathrm{d} \varphi(X, Y)=\nabla_{X}^{\varphi}(\mathrm{d} \varphi(Y))-\mathrm{d} \varphi\left(\nabla_{X}^{M} Y\right)=B(X, Y),
$$

that is, the second fundamental form $\nabla \mathrm{d} \varphi(X, Y)$ of the isometric immersion $\varphi$ agrees with the second fundamental form $B(X, Y)$ of the immersed submanifold $\varphi(M)$ in $N$; for details see [Kobayashi and Nomizu 1969, Chapter 7] and [Baird and Wood 2003, Example 3.2.3]. From (2) we see that the tension field $\tau(\varphi)$ of an isometric immersion and the mean curvature vector field $\eta$ of the submanifold are related by

$$
\tau(\varphi)=m \eta
$$

For a hypersurface, that is, a codimension one isometric immersion $\varphi$ of $M^{m}$ into $N^{m+1}$, we can choose a local unit vector field $\xi$ normal to $\varphi(M) \subset N$. Then $\eta=H \xi$ for $H$ the mean curvature function, and we can write $B(X, Y)=b(X, Y) \xi$, where $b: T M \times T M \rightarrow C^{\infty}(M)$ is the second fundamental form. The relationship between the shape operator $A$ of the hypersurface with respect to the unit normal vector field $\xi$ and the second fundamental form is given by

$$
\begin{aligned}
& B(X, Y)=\left\langle\nabla_{X}^{N} Y, \xi\right\rangle \xi=-\left\langle Y, \nabla_{X}^{N} \xi\right\rangle \xi=\langle A X, Y\rangle \xi, \\
& \langle A X, Y\rangle=\langle B(X, Y), \xi\rangle=\langle b(X, Y) \xi, \xi\rangle=b(X, Y) .
\end{aligned}
$$

Theorem 2.1. Let $\varphi: M^{m} \rightarrow N^{m+1}$ be an isometric immersion of codimension one with mean curvature vector $\eta=H \xi$. Then $\varphi$ is biharmonic if and only if

$$
\Delta H-H|A|^{2}+H \operatorname{Ric}^{N}(\xi, \xi)=0,
$$

$$
2 A(\operatorname{grad} H)+\frac{1}{2} m \operatorname{grad} H^{2}-2 H\left(\operatorname{Ric}^{N}(\xi)\right)^{\top}=0,
$$

where $\operatorname{Ric}^{N}: T_{q} N \rightarrow T_{q} N$ denotes the Ricci operator of the ambient space, defined by $\left\langle\operatorname{Ric}^{N}(Z), W\right\rangle=\operatorname{Ric}^{N}(Z, W)$, and $A$ is the shape operator of the hypersurface with respect to the unit normal vector $\xi$. 
Proof. First choose a local orthonormal frame $\left\{e_{i}\right\}_{i=1, \ldots, m}$ on $M$ such that the orthonormal frame $\left\{\mathrm{d} \varphi\left(e_{1}\right), \ldots, \mathrm{d} \varphi\left(e_{m}\right), \xi\right\}$ is adapted to the ambient space defined on the hypersurface. Identifying $\mathrm{d} \varphi(X)=X$ and $\nabla_{X}^{\varphi} W=\nabla_{X}^{N} W$ and noting that the tension field of $\varphi$ is $\tau(\varphi)=m H \xi$, we can compute the bitension field of $\varphi$ as

$$
\begin{aligned}
& \tau^{2}(\varphi)= \sum_{i=1}^{m}\left\{\nabla_{e_{i}}^{\varphi} \nabla_{e_{i}}^{\varphi}(m H \xi)-\nabla_{\nabla_{e_{i}} e_{i}}^{\varphi}(m H \xi)-R^{N}\left(\mathrm{~d} \varphi\left(e_{i}\right), m H \xi\right) d \varphi\left(e_{i}\right)\right\} \\
&=m \sum_{i=1}^{m}\left(e_{i} e_{i}(H) \xi+2 e_{i}(H) \nabla_{e_{i}}^{N} \xi+H \nabla_{e_{i}}^{N} \nabla_{e_{i}}^{N} \xi-\left(\nabla_{e_{i}} e_{i}\right)(H) \xi-H \nabla_{\nabla_{e_{i}} e_{i}}^{N} \xi\right) \\
&-m H \sum_{i=1}^{m} R^{N}\left(\mathrm{~d} \varphi\left(e_{i}\right), \xi\right) d \varphi\left(e_{i}\right) \\
&=m(\Delta H) \xi-2 m A(\operatorname{grad} H)-m H \Delta^{\varphi} \xi-m H \sum_{i=1}^{m} R^{N}\left(\mathrm{~d} \varphi\left(e_{i}\right), \xi\right) d \varphi\left(e_{i}\right) .
\end{aligned}
$$

To find the tangential and normal parts of the bitension field, we first compute the tangential and normal components of the curvature term, getting

$$
\begin{aligned}
& \sum_{i, k=1}^{m}\left\langle R^{N}\left(\mathrm{~d} \varphi\left(e_{i}\right), \xi\right) d \varphi\left(e_{i}\right), e_{k}\right\rangle e_{k}=-\left(\operatorname{Ric}^{N}\left(\xi, e_{k}\right)\right) e_{k}=-(\operatorname{Ric}(\xi))^{\top}, \\
& \sum_{i=1}^{m}\left\langle R^{N}\left(\mathrm{~d} \varphi\left(e_{i}\right), \tau(\varphi)\right) d \varphi\left(e_{i}\right), \xi\right\rangle=-m H \operatorname{Ric}^{N}(\xi, \xi) .
\end{aligned}
$$

To find the normal part of $\Delta^{\varphi} \xi$, we compute

$$
\left\langle\Delta^{\varphi} \xi, \xi\right\rangle=\sum_{i=1}^{m}\left\langle-\nabla_{e_{i}}^{N} \nabla_{e_{i}}^{N} \xi+\nabla_{\nabla_{e_{i}} e_{i}}^{N} \xi, \xi\right\rangle=\sum_{i=1}^{m}\left\langle\nabla_{e_{i}}^{N} \xi, \nabla_{e_{i}}^{N} \xi\right\rangle
$$

On the other hand, using (3) and (4), we have

$$
\begin{aligned}
|A|^{2} & =\sum_{i, j=1}^{m}\left\langle A e_{i}, e_{j}\right\rangle^{2}=\sum_{i, j=1}^{m}\left\langle\nabla_{e_{i}}^{N} \xi, e_{j}\right\rangle^{2}=\sum_{i=1}^{m}\left\langle\nabla_{e_{i}}^{N} \xi, \sum_{j=1}^{m}\left\langle\nabla_{e_{i}}^{N} \xi, e_{j}\right\rangle e_{j}\right\rangle \\
& =\sum_{i=1}^{m}\left\langle\nabla_{e_{i}}^{N} \xi, \nabla_{e_{i}}^{N} \xi\right\rangle
\end{aligned}
$$

which, together with (6), implies that

$$
\left(\Delta^{\varphi} \xi\right)^{\perp}=\left\langle\Delta^{\varphi} \xi, \xi\right\rangle \xi=\sum_{i=1}^{m}\left\langle\nabla_{e_{i}}^{N} \xi, \nabla_{e_{i}}^{N} \xi\right\rangle \xi=|A|^{2} \xi .
$$


A straightforward computation gives the tangential part of $\Delta^{\varphi} \xi$ as

$$
\begin{aligned}
\left(\Delta^{\varphi} \xi\right)^{\top} & =\sum_{i, k=1}^{m}\left\langle-\nabla_{e_{i}}^{N} \nabla_{e_{i}}^{N} \xi+\nabla_{\nabla_{e_{i}} e_{i}}^{N} \xi, e_{k}\right\rangle e_{k} \\
& =\sum_{i, k=1}^{m}\left\langle\nabla_{e_{i}}^{N} A e_{i}-A\left(\nabla_{e_{i}} e_{i}\right), e_{k}\right\rangle e_{k}=\sum_{i, k=1}^{m}\left(\left(\nabla_{e_{i}} b\right)\left(e_{k}, e_{i}\right)\right) e_{k} .
\end{aligned}
$$

Substituting the Codazzi-Mainardi equation for a hypersurface, namely,

$$
\left(\nabla_{e_{i}} b\right)\left(e_{k}, e_{i}\right)-\left(\nabla_{e_{k}} b\right)\left(e_{i}, e_{i}\right)=\left(R^{N}\left(e_{i}, e_{k}\right) e_{i}\right)^{\perp}=\left\langle R^{N}\left(e_{i}, e_{k}\right) e_{i}, \xi\right\rangle,
$$

into (7) and using normal coordinates at a point, we have

$$
\begin{aligned}
\left(\Delta^{\varphi} \xi\right)^{\top} & =\sum_{i, k=1}^{m}\left(\left(\nabla_{e_{i}} b\right)\left(e_{k} e_{i}\right)\right) e_{k} \\
& =\sum_{k=1}^{m}\left(\sum_{i=1}^{m}\left(\nabla_{e_{k}} b\right)\left(e_{i}, e_{i}\right)-\operatorname{Ric}\left(\xi, e_{k}\right)\right) e_{k}=m \operatorname{grad}(H)-\left(\operatorname{Ric}\left(\xi, e_{k}\right)\right) e_{k} .
\end{aligned}
$$

Therefore, by collecting all the tangent and normal parts of the bitension field separately, we finally have

$$
\begin{aligned}
\left(\tau^{2}(\varphi)\right)^{\perp} & =\left\langle\tau^{2}(\varphi), \xi\right\rangle \xi=m\left(\Delta H-H|A|^{2}+H \operatorname{Ric}^{N}(\xi, \xi)\right) \xi, \\
\left(\tau^{2}(\varphi)\right)^{\top} & =\sum_{k=1}^{m}\left\langle\tau^{2}(\varphi), e_{k}\right\rangle e_{k} \\
& =-m\left(2 A(\operatorname{grad} H)+\frac{1}{2} m\left(\operatorname{grad} H^{2}\right)-2 H(\operatorname{Ric}(\xi))^{\top}\right) .
\end{aligned}
$$

As an immediate consequence of Theorem 2.1 is this:

Corollary 2.2. A constant mean curvature hypersurface in a Riemannian manifold is biharmonic if and only if it is minimal or $\operatorname{Ric}^{N}(\xi, \xi)=|A|^{2}$ and $\left(\operatorname{Ric}^{N}(\xi)\right)^{\top}=0$. In particular, we recover [Oniciuc 2002, Proposition 2.4], which states that a constant mean curvature hypersurface in a Riemannian manifold $\left(N^{m+1}, h\right)$ with nonpositive Ricci curvature is biharmonic if and only if it is minimal.

Corollary 2.3. A hypersurface in an Einstein space $\left(N^{m+1}, h\right)$ is biharmonic if and only if its mean curvature function $H$ is a solution of the PDEs

$$
\begin{aligned}
\Delta H-H|A|^{2}+\frac{r H}{m+1} & =0, \\
2 A(\operatorname{grad} H)+\frac{1}{2} m \operatorname{grad} H^{2} & =0,
\end{aligned}
$$

where $r$ is the scalar curvature of the ambient space. In particular, a hypersurface $\varphi:\left(M^{m}, g\right) \rightarrow\left(N^{m+1}(C), h\right)$ in a space of constant sectional curvature $C$ is 
biharmonic if and only if its mean curvature function $H$ is a solution of the PDEs [Jiang 1987; Chen 1991; Caddeo et al. 2002]

$$
\begin{aligned}
\Delta H-H|A|^{2}+m C H & =0, \\
2 A(\operatorname{grad} H)+\frac{1}{2} m \operatorname{grad} H^{2} & =0 .
\end{aligned}
$$

Proof. It is well known that if $\left(N^{m+1}, h\right)$ is an Einstein manifold, then

$$
\operatorname{Ric}^{N}(Z, W)=\frac{r}{m+1} h(Z, W) \text { for any } Z, W \in T N
$$

and hence $\left(\operatorname{Ric}^{N}(\xi)\right)^{\top}=0$ and $\operatorname{Ric}^{N}(\xi, \xi)=r /(m+1)$. From these and (5) we obtain (8). When $\left(N^{m+1}(C), h\right)$ is a space of constant sectional curvature $C$, it is an Einstein space with scalar curvature $r=m(m+1) C$. Substituting this into (8) we obtain (9).

Theorem 2.4. A totally umbilical hypersurface in an Einstein space with nonpositive scalar curvature is biharmonic if and only if it is minimal.

Proof. Take an orthonormal frame $\left\{e_{1}, \ldots, e_{m}, \xi\right\}$ of $\left(N^{m+1}, h\right)$ adapted to the hypersurface $M$ so that $A e_{i}=\lambda_{i} e_{i}$, where $A$ is the Weingarten map of the hypersurface and $\lambda_{i}$ is the principal curvature in the direction $e_{i}$. Since $M$ is assumed to be totally umbilical, all principal normal curvatures at any point $p \in M$ are equal to the same number $\lambda(p)$. It follows that

$$
\begin{aligned}
H & =\frac{1}{m} \sum_{i=1}^{m}\left\langle A e_{i}, e_{i}\right\rangle=\lambda, \quad|A|^{2}=m \lambda^{2}, \\
A(\operatorname{grad} H) & =A\left(\sum_{i=1}^{m}\left(e_{i} \lambda\right) e_{i}\right)=\frac{1}{2} \operatorname{grad} \lambda^{2},
\end{aligned}
$$

The biharmonic hypersurface equations (8) become

$$
\Delta \lambda-m \lambda^{3}+\frac{r \lambda}{m+1}=0 \quad \text { and } \quad(2+m) \operatorname{grad} \lambda^{2}=0 .
$$

Solving these, we have either $\lambda=0$ and hence $H=0$, or $\lambda= \pm \sqrt{r /(m(m+1))}$ is a constant. The latter happens only if the scalar curvature is nonnegative, from which we obtain the theorem.

Remark 2.5. Theorem 2.4 generalizes the results of [Balmuş et al. 2008; Caddeo et al. 2002; Dimitrić 1992] about totally umbilical biharmonic hypersurfaces in a space form. It also implies that the generalized Chen conjecture is true for totally umbilical hypersurfaces in an Einstein space with nonpositive scalar curvature. Note that nonpositive scalar curvature is a much weaker condition than nonpositive sectional curvature. 
Corollary 2.6. Any totally umbilical biharmonic hypersurface in a Ricci flat manifold is minimal.

Proof. This follows from Theorem 2.4 and the fact that a Ricci flat manifold is an Einstein space with zero scalar curvature.

\section{Proper biharmonic foliations of codimension one}

In general, proper biharmonic maps as local solutions of a system of fourth order PDEs are extremely difficult to unearth. Even in the case of biharmonic submanifolds (viewed as biharmonic maps with geometric constraints), few examples have been found. In this section, we construct families of metrics that turn some foliations of hypersurfaces into proper biharmonic foliations, thus providing infinitely many proper biharmonic hypersurfaces.

Theorem 3.1. For any constant $C$, let $N=\left\{\left(x_{1}, \ldots, x_{m}, z\right) \in \mathbb{R}^{m+1} \mid z>-C\right\}$ denote the upper half space. Then, the conformally flat space

$$
\left(N, h=f^{-2}(z)\left(\sum_{i=1}^{m} \mathrm{~d} x_{i}^{2}+\mathrm{d} z^{2}\right)\right)
$$

is foliated by proper biharmonic hyperplanes $z=k$, where $k \in \mathbb{R}$ and $k>-C$, if and only if $f(z)=D /(z+E)$, where $E \geq C$ and $D \in \mathbb{R} \backslash\{0\}$.

Proof. Consider the isometric immersion

$$
\varphi:\left(\mathbb{R}^{m}, g\right) \rightarrow\left(\mathbb{R}^{m+1}, h=f^{-2}(z)\left(\sum_{i=1}^{m} \mathrm{~d} x_{i}{ }^{2}+\mathrm{d} z^{2}\right)\right)
$$

with $\varphi\left(x_{1}, \ldots, x_{m}\right)=\left(x_{1}, \ldots, x_{m}, k\right)$ and $k$ being a constant, where the induced metric $g$ with respect to the natural frame $\partial_{i}=\partial / \partial x_{i}$ for $i=1,2, \ldots, m$ and $\partial_{m+1}=\partial / \partial z$ has components

$$
g_{i j}=g\left(\partial_{i}, \partial_{j}\right)=h\left(d \varphi\left(\partial_{i}\right), d \varphi\left(\partial_{j}\right)\right) \circ \varphi= \begin{cases}f^{-2}(k) & \text { if } i=j, \\ 0 & \text { if } i \neq j .\end{cases}
$$

One can check that $e_{A}=f(z) \partial_{A}$, where $A=1,2, \ldots, m, m+1$, constitutes a local orthonormal frame on $\mathbb{R}^{m+1}$ adapted to the hypersurface $z=k$ with $\xi=e_{m+1}$ being the unit normal vector field. A straightforward computation using Koszul's formula gives the coefficients of the Levi-Civita connection of the ambient space:

$$
\left(\nabla_{e_{A}} e_{B}\right)=\left(\begin{array}{ccccc}
f^{\prime} e_{m+1} & 0 & \cdots & 0 & -f^{\prime} e_{1} \\
0 & f^{\prime} e_{m+1} & \cdots & 0 & -f^{\prime} e_{2} \\
\vdots & \vdots & \ddots & \vdots & \vdots \\
0 & 0 & \cdots & f^{\prime} e_{m+1} & -f^{\prime} e_{m} \\
0 & 0 & \cdots & 0 & 0
\end{array}\right)_{(m+1) \times(m+1)}
$$


Noting that $\xi=e_{m+1}$ is the unit normal vector field, we can easily compute the components of the second fundamental form as

$$
h\left(e_{i}, e_{j}\right)=\left\langle\nabla_{e_{i}} e_{j}, e_{m+1}\right\rangle= \begin{cases}f^{\prime} & \text { if } i=j=1,2, \ldots, m \\ 0 & \text { otherwise }\end{cases}
$$

from which we conclude that each of the hyperplanes $z=k$ is a totally umbilical hypersurface in the conformally flat space.

We compute the mean curvature of the hypersurface and the norm of the second fundamental form to be

$$
H=\frac{1}{m} \sum_{i=1}^{m} h\left(e_{i}, e_{i}\right)=f^{\prime} \quad \text { and } \quad|A|^{2}=\sum_{i=1}^{m}\left|h\left(e_{i}, e_{i}\right)\right|^{2}=m f^{\prime 2} .
$$

Since $H$ depends only on $z$, we have $\operatorname{grad}_{g} H=\sum_{i=1}^{m} e_{i}(H) e_{i}=0$ and hence $\Delta_{g} H=\operatorname{div}\left(\operatorname{grad}_{g} H\right)=0$. Therefore, by Theorem 2.1, the biharmonic equation of the isometric immersion reduces to the system

$$
-|A|^{2}+\operatorname{Ric}^{N}(\xi, \xi)=0 \quad \text { and } \quad \sum_{i=1}^{m}\left(\operatorname{Ric}^{N}\left(\xi, e_{i}\right)\right) e_{i}=0 .
$$

We can compute the Ricci curvature of the ambient space:

$\operatorname{Ric}\left(e_{i}, \xi\right)=\operatorname{Ric}\left(e_{i}, e_{m+1}\right)=\sum_{j=1}^{m}\left\langle R\left(e_{m+1}, e_{j}\right) e_{j}, e_{i}\right\rangle=0 \quad$ for all $i=1,2, \ldots, m$, $\operatorname{Ric}(\xi, \xi)=\operatorname{Ric}\left(e_{m+1}, e_{m+1}\right)=\sum_{j=1}^{m}\left\langle R\left(e_{m+1}, e_{j}\right) e_{j}, e_{m+1}\right\rangle=m f f^{\prime \prime}-m f^{\prime 2}$.

Substituting these into the system (10), we conclude that all isometric immersions $\varphi: \mathbb{R}^{m} \rightarrow\left(\mathbb{R}^{m+1}, h=f^{-2}(z)\left(\sum_{i=1}^{m} \mathrm{~d} x_{i}{ }^{2}+\mathrm{d} z^{2}\right)\right)$ with $\varphi\left(x_{1}, \ldots, x_{m}\right)=$ $\left(x_{1}, \ldots, x_{m}, k\right)$ are biharmonic if and only if $f f^{\prime \prime}-2 f^{\prime 2}=0$. This equation can be written as $\left(f^{\prime} / f\right)^{\prime}-\left(f^{\prime} / f\right)^{2}=0$. This ordinary differential equation has solution $f(z)=D /(z+C)$, where $C$ and $D$ are constants. Since the mean curvature of the hypersurface $H=f^{\prime}(k)$ is never zero, we conclude that each of the hyperplanes $z=k$ for $k \neq-C$ is a proper biharmonic hypersurface in the conformally flat space $\left(N, h=((z+C) / D)^{2}\left(\sum_{i=1}^{m} \mathrm{~d} x_{i}{ }^{2}+\mathrm{d} z^{2}\right)\right)$.

Theorem 3.2. The isometric immersion

$$
\varphi: \mathbb{R}^{2} \rightarrow\left(\mathbb{R}^{3}, h=e^{2 p(z)} \mathrm{d} x^{2}+e^{2 q(z)} \mathrm{d} y^{2}+\mathrm{d} z^{2}\right)
$$

with $\varphi(x, y)=(x, y, c)$ is biharmonic if and only if

$$
p^{\prime \prime}+2 p^{\prime 2}+q^{\prime \prime}+2 q^{\prime 2}=0 .
$$


In particular, for any positive constants $A, B, C, D$, the upper half space $\mathbb{R}_{+}^{3}=$ $\{(x, y, z) \mid z>0\}$ with metric $h=(A z+B) \mathrm{d} x^{2}+(C z+D) \mathrm{d} y^{2}+\mathrm{d} z^{2}$ is foliated by proper biharmonic planes $z=$ constant.

Proof. Let $\varphi$ be as stated, with $c$ being a positive constant. Using the notation $\partial_{1}=\partial / \partial x, \partial_{2}=\partial / \partial y$ and $\partial_{3}=\partial / \partial z$ we can easily check that the induced metric is given by

$$
\begin{aligned}
& g_{11}=g\left(\partial_{1}, \partial_{1}\right)=h\left(d \varphi\left(\partial_{1}\right), d \varphi\left(\partial_{1}\right)\right) \circ \varphi=e^{2 p(c)}, \\
& g_{12}=g\left(\partial_{1}, \partial_{2}\right)=h\left(d \varphi\left(\partial_{1}\right), d \varphi\left(\partial_{2}\right)\right) \circ \varphi=0, \\
& g_{22}=g\left(\partial_{2}, \partial_{2}\right)=h\left(d \varphi\left(\partial_{2}\right), d \varphi\left(\partial_{2}\right)\right) \circ \varphi=e^{2 q(c)} .
\end{aligned}
$$

One can also check that $e_{1}=e^{-p(z)} \partial_{1}, e_{2}=e^{-q(z)} \partial_{2}$ and $e_{3}=\partial_{3}$ constitute an orthonormal frame on $\mathbb{R}_{+}^{3}$ adapted to the surface $z=c$, with $\xi=e_{3}$ being the unit normal vector field. A further computation gives the Lie brackets

$$
\left[e_{1}, e_{2}\right]=0, \quad\left[e_{1}, e_{3}\right]=p^{\prime} e_{1}, \quad\left[e_{2}, e_{3}\right]=q^{\prime} e_{2},
$$

and the coefficients of the Levi-Civita connection:

$$
\begin{array}{lll}
\nabla_{e_{1}} e_{1}=-p^{\prime} e_{3}, & \nabla_{e_{1}} e_{2}=0, & \nabla_{e_{1}} e_{3}=p^{\prime} e_{1}, \\
\nabla_{e_{2}} e_{1}=0, & \nabla_{e_{2}} e_{2}=-q^{\prime} e_{3}, & \nabla_{e_{2}} e_{3}=q^{\prime} e_{2}, \\
\nabla_{e_{3}} e_{1}=0, & \nabla_{e_{3}} e_{2}=0, & \nabla_{e_{3}} e_{3}=0 .
\end{array}
$$

Since that $\xi=e_{3}$ is the unit normal vector field, the components of the second fundamental form are

$$
\begin{aligned}
& h\left(e_{1}, e_{1}\right)=\left\langle\nabla_{e_{1}} e_{1}, e_{3}\right\rangle=-p^{\prime}, \\
& h\left(e_{1}, e_{2}\right)=\left\langle\nabla_{e_{1}} e_{2}, e_{3}\right\rangle=0, \\
& h\left(e_{2}, e_{2}\right)=\left\langle\nabla_{e_{2}} e_{2}, e_{3}\right\rangle=-q^{\prime} .
\end{aligned}
$$

From these, the mean curvature of the isometric immersion is

$$
H=\frac{1}{2}\left(h\left(e_{1}, e_{1}\right)+h\left(e_{2}, e_{2}\right)\right)=-\left(p^{\prime}+q^{\prime}\right) / 2,
$$

and the norm of the second fundamental form is

$$
|A|^{2}=\sum_{i=1}^{2}\left|h\left(e_{i}, e_{i}\right)\right|^{2}=p^{\prime 2}+q^{\prime 2} .
$$

Since $H$ depends only on $z$ we have $\operatorname{grad}_{g} H=e_{1}(H) e_{1}+e_{2}(H) e_{2}=0$ and hence $\Delta_{g} H=\operatorname{div}\left(\operatorname{grad}_{g} H\right)=0$. Therefore, by Theorem 2.1, the biharmonic equation of the isometric immersion reduces to (10) with $m=2$. To compute the Ricci 
curvature of the ambient space we can use (12) and (13) to get

$$
\begin{aligned}
\operatorname{Ric}\left(e_{1}, \xi\right)=\operatorname{Ric}\left(e_{1}, e_{3}\right) & =\left\langle R\left(e_{3}, e_{2}\right) e_{2}, e_{1}\right\rangle=0, \\
\operatorname{Ric}\left(e_{2}, \xi\right)=\operatorname{Ric}\left(e_{2}, e_{3}\right) & =\left\langle R\left(e_{3}, e_{1}\right) e_{1}, e_{3}\right\rangle=0, \\
\operatorname{Ric}(\xi, \xi)=\operatorname{Ric}\left(e_{3}, e_{3}\right) & =\left\langle R\left(e_{3}, e_{1}\right) e_{1}, e_{3}\right\rangle+\left\langle R\left(e_{3}, e_{2}\right) e_{2}, e_{3}\right\rangle \\
& =-p^{\prime \prime}-p^{\prime 2}-q^{\prime \prime}-q^{\prime 2} .
\end{aligned}
$$

Substituting these into (10) with $m=2$, we conclude that $\varphi$ is biharmonic if and only if (11) holds, which gives the theorem's first statement. The second is obtained by looking for the solutions of (11) satisfying $p^{\prime \prime}+2 p^{\prime 2}=0$ and $q^{\prime \prime}+2 q^{\prime 2}=0$. In fact, we have special solutions $p(z)=\frac{1}{2} \ln (A z+B)$ and $q(z)=\frac{1}{2} \ln (C z+D)$ with positive constants $A, B, C, D$. By (14) and the choice of these constants, we see that the mean curvature of the surface $z=c$ is

$$
H=-\frac{2 A C z+A D+B C}{2(A z+B)(C z+D)} \neq 0 \text {, }
$$

and hence each such surface is a nonminimal biharmonic surface.

Remark 3.3. Theorem 3.2 has a generalization to a higher dimensional space $\mathbb{R}_{+}^{m}$ for $m>3$.

Example 3.4. Let $\lambda(t)=\sqrt{A t+B}$, where $A$ and $B$ are positive constants. Then the warped product space $N=\left(S^{2} \times \mathbb{R}^{+}, h=\lambda^{2}(t) g^{S^{2}}+\mathrm{d} t^{2}\right)$ is foliated by the spheres $\left(S^{2} \times\{t\}, \lambda^{2}(t) g^{S^{2}}\right)$, each of which is a totally umbilical proper biharmonic surface.

To see what is claimed in Example 3.4, we parametrize the unit sphere $S^{2}$ by spherical polar coordinates:

$$
\mathbb{R} \times \mathbb{R} \ni(\rho, \theta) \rightarrow(\cos \rho, \sin \rho \cos \theta, \sin \rho \sin \theta)) \in \mathbb{R}^{3} .
$$

Then, the standard metric can be written as $g^{S^{2}}=\mathrm{d} \rho^{2}+\sin ^{2} \rho \mathrm{d} \theta^{2}$, and the warped product metric on $N$ takes the form $h=\lambda^{2}(t) \mathrm{d} \rho^{2}+\lambda^{2}(t) \sin ^{2} \rho \mathrm{d} \theta^{2}+\mathrm{d} t^{2}$. Consider the isometric immersion $\varphi: S^{2} \rightarrow\left(\mathbb{R}^{+} \times S^{2}, \mathrm{~d} t^{2}+\lambda^{2}(t) g^{2}\right)$ with $\varphi(\rho, \theta)=(\rho, \theta, c)$ and $c$ being a positive constant. Using the notation $\partial_{1}=\partial / \partial \rho, \partial_{2}=\partial / \partial \theta$ and $\partial_{3}=\partial / \partial t$, we can easily check that the induced metric is given by

$$
\begin{aligned}
& g_{11}=g\left(\partial_{1}, \partial_{1}\right)=h\left(d \varphi\left(\partial_{1}\right), d \varphi\left(\partial_{1}\right)\right) \circ \varphi=\lambda^{2}(c), \\
& g_{12}=g\left(\partial_{1}, \partial_{2}\right)=h\left(d \varphi\left(\partial_{1}\right), d \varphi\left(\partial_{2}\right)\right) \circ \varphi=0, \\
& g_{22}=g\left(\partial_{2}, \partial_{2}\right)=h\left(d \varphi\left(\partial_{2}\right), d \varphi\left(\partial_{2}\right)\right) \circ \varphi=\lambda^{2}(c) \sin ^{2} \rho .
\end{aligned}
$$

Using the orthonormal frame $e_{1}=\lambda^{-1}(t) \partial_{1}, e_{2}=(\lambda(t) \sin \rho)^{-1} \partial_{2}$ and $e_{3}=\partial_{3}$, we have the Lie brackets

$$
\left[e_{1}, e_{2}\right]=-(\cot \rho / \lambda) e_{2}, \quad\left[e_{1}, e_{3}\right]=f e_{1}, \quad\left[e_{2}, e_{3}\right]=f e_{2},
$$


where here and in the sequel we use the notation $f=(\ln \lambda)^{\prime}=\lambda^{\prime} / \lambda$. Clearly, $e_{1}, e_{2}$ and $\xi=e_{3}=\partial_{3}$ constitute a local orthonormal frame of $N$ adapted to the surface with $\xi$ being the unit vector field normal to the surface. We can use the Kozsul formula to compute the components of the second fundamental form as

$$
\begin{aligned}
h\left(e_{1}, e_{1}\right) & =\left\langle\nabla_{e_{1}} e_{1}, \xi\right\rangle=\left\langle\nabla_{e_{1}} e_{1}, e_{3}\right\rangle \\
& =\frac{1}{2}\left(-\left\langle e_{1},\left[e_{1}, e_{3}\right]\right\rangle-\left\langle e_{1},\left[e_{1}, e_{3}\right]\right\rangle+\left\langle e_{3},\left[e_{1}, e_{1}\right]\right\rangle\right)=-f, \\
h\left(e_{1}, e_{2}\right) & =\left\langle\nabla_{e_{1}} e_{2}, \xi\right\rangle=\left\langle\nabla_{e_{1}} e_{2}, e_{3}\right\rangle=0, \\
h\left(e_{2}, e_{2}\right) & =\left\langle\nabla_{e_{2}} e_{2}, \xi\right\rangle=\left\langle\nabla_{e_{2}} e_{2}, e_{3}\right\rangle=-f,
\end{aligned}
$$

from which we conclude that each such sphere is a totally umbilical surface in $N$.

The mean curvature of the isometric immersion and the norm of the second fundamental form are

$$
H=\frac{1}{2}\left(h\left(e_{1}, e_{1}\right)+h\left(e_{2}, e_{2}\right)\right)=-f \quad \text { and } \quad|A|^{2}=\sum_{i=1}^{2}\left|h\left(e_{i}, e_{i}\right)\right|^{2}=2 f^{2},
$$

which depend only on $t$. It follows that $\operatorname{grad}_{g} H=0$ and $\Delta_{g} H=0$. Therefore, by Theorem 2.1, the proper biharmonic equation of $\varphi$ reduces to (10) with $m=2$.

On the other hand, using the Ricci curvature formula (for example [Besse 2008]) of the warped product $M=B \times_{\lambda} F$, we have

$$
\begin{aligned}
\operatorname{Ric}\left(e_{1}, \xi\right) & =\operatorname{Ric}\left(e_{1}, e_{3}\right)=0, \quad \operatorname{Ric}\left(e_{2}, \xi\right)=\operatorname{Ric}\left(e_{2}, e_{3}\right)=0, \\
\operatorname{Ric}(\xi, \xi) & =\operatorname{Ric}\left(e_{3}, e_{3}\right)=\operatorname{Ric}^{\mathbb{R}}\left(e_{3}, e_{3}\right)-(2 / \lambda) \operatorname{Hess}_{\lambda}\left(e_{3}, e_{3}\right) \\
& =-(2 / \lambda)\left(e_{3}\left(e_{3} \lambda\right)-\mathrm{d} \lambda\left(\nabla_{e_{3}} e_{3}\right)\right)=-2 \lambda^{\prime \prime} / \lambda .
\end{aligned}
$$

Substituting these into (10) with $m=2$ we conclude that the isometric immersion $\varphi: S^{2} \rightarrow\left(S^{2} \times \mathbb{R}^{+}, \lambda^{2}(t) g^{S^{2}}+\mathrm{d} t^{2}\right)$ with $\varphi(\rho, \theta)=(\rho, \theta, c)$ is biharmonic if and only if $-2\left(\lambda^{\prime} / \lambda\right)^{2}-2 \lambda^{\prime \prime} / \lambda=0$. Solving this final equation, we have $\lambda(t)=\sqrt{A t+B}$, proving the claim in Example 3.4.

Remark 3.5. The referee points out that the biharmonicity of the inclusion maps in Example 3.4 is in fact a special case of [Balmuş et al. 2007, Corollary 3.4], which was proved by a different method.

\section{Biharmonic cylinders of a Riemannian submersion}

Let $\pi:\left(M^{3}, g\right) \rightarrow\left(N^{2}, h\right)$ be a Riemannian submersion with totally geodesic fibers from a complete manifold. Let $\alpha: I \rightarrow\left(N^{2}, h\right)$ be an immersed regular curve parametrized by arclength. Then $\Sigma=\bigcup_{t \in I} \pi^{-1}(\alpha(t))$ is a surface in $M$ that can be viewed as a disjoint union of all horizontal lifts of the curve $\alpha$. Let $\left\{\bar{X}=\alpha^{\prime}, \bar{\xi}\right\}$ be a Frenet frame along $\alpha$, and let $\bar{\kappa}$ be the geodesic curvature of the 
curve. Then the Frenet formula for $\alpha$ is given by

$$
\begin{aligned}
& \tilde{\nabla}_{\bar{X}} \bar{X}=\bar{\kappa} \bar{\xi}, \\
& \tilde{\nabla}_{\bar{X}} \bar{\xi}=-\bar{\kappa} \bar{X},
\end{aligned}
$$

where $\tilde{\nabla}$ denotes the Levi-Civita connection of $(N, h)$. Let $\beta: I \rightarrow\left(M^{3}, g\right)$ be a horizontal lift of $\alpha$. Let $X$ and $\xi$ be the horizontal lifts of $\bar{X}$ and $\bar{\xi}$, respectively. Let $V$ be the unit vector field tangent to the fibers of the submersion $\pi$. Then $\{X, \xi, V\}$ is an orthonormal frame of $M$ adapted to the surface, with $\xi$ the unit normal vector of the surface. The restriction of this frame to the curve $\beta$ is the Frenet frame along $\beta$. Therefore, the Frenet formula along $\beta$ is given by

$$
\begin{aligned}
\nabla_{X} X & =\kappa \xi, \\
\nabla_{X} \xi & =-\kappa X+\tau V, \\
\nabla_{X} V & =-\tau \xi,
\end{aligned}
$$

where $\nabla$ denotes the Levi-Civita connection of $(M, g)$. Since a Riemannian submersion preserves the inner product of horizontal vector fields, we can check that $\kappa=\bar{\kappa} \circ \pi$ and $\tau=\left\langle\nabla_{X} \xi, V\right\rangle=\left\langle A_{X} \xi, V\right\rangle$ is the torsion of the horizontal lift that vanishes if the Riemannian submersion has integrable horizontal distribution; here $A$ is the $A$-tensor of the submersion [O'Neill 1966]. In what follows, we will use the frame $\{X, \xi, V\}$ to compute the mean curvature, second fundamental form, and other terms that appear in the biharmonic equation of the surface $\Sigma$.

Using (15) we have

$$
\begin{aligned}
A(X) & =-\left\langle\nabla_{X} \xi, X\right\rangle X-\left\langle\nabla_{X} \xi, V\right\rangle V=\kappa X-\tau V, \\
A(V) & =-\left\langle\nabla_{V} \xi, X\right\rangle X-\left\langle\nabla_{V} \xi, V\right\rangle V=-\tau X, \\
b(X, X) & =\langle A(X), X\rangle=\kappa, \quad b(X, V)=\langle A(X), V\rangle=-\tau, \\
b(V, X) & =\langle A(V), X\rangle=-\tau, \quad b(V, V)=\langle A(V), V\rangle=0 ; \\
H & =\frac{1}{2}(b(X, X)+b(V, V))=\frac{1}{2} \kappa, \\
A(\operatorname{grad} H) & =A\left(X\left(\frac{1}{2} \kappa\right) X+V\left(\frac{1}{2} \kappa\right) V\right)=X\left(\frac{1}{2} \kappa\right) A(X)=\frac{1}{2} \kappa^{\prime}(\kappa X-\tau V) ; \\
\Delta H & =X X(H)-\left(\nabla_{X} X\right) H+V V(H)-\left(\nabla_{V} V\right) H=\frac{1}{2} \kappa^{\prime \prime} ; \\
|A|^{2} & =(b(X, X))^{2}+(b(X, V))^{2}+(b(V, X))^{2}+(b(V, V))^{2}=\kappa^{2}+2 \tau^{2} .
\end{aligned}
$$

Substituting these into the biharmonic hypersurface equation (5), we conclude that the surface $\Sigma$ is biharmonic in $\left(M^{3}, g\right)$ if and only if

$$
\begin{aligned}
\frac{1}{2} \kappa^{\prime \prime}-\frac{1}{2} \kappa\left(\kappa^{2}+2 \tau^{2}\right)+\frac{1}{2} \kappa \operatorname{Ric}^{M}(\xi, \xi) & =0, \\
\kappa^{\prime}(\kappa X-\tau V)+\frac{1}{2} \kappa \kappa^{\prime} X-\kappa \operatorname{Ric}^{M}(\xi, X) X-\kappa \operatorname{Ric}^{M}(\xi, V) V & =0 .
\end{aligned}
$$


These are equivalent to

$$
\begin{aligned}
\kappa^{\prime \prime}-\kappa\left(\kappa^{2}+2 \tau^{2}\right)+\kappa \operatorname{Ric}^{M}(\xi, \xi) & =0, \\
3 \kappa^{\prime} \kappa-2 \kappa \operatorname{Ric}^{M}(\xi, X) & =0, \\
\kappa^{\prime} \tau+\kappa \operatorname{Ric}^{M}(\xi, V) & =0 .
\end{aligned}
$$

Applying (16) to Hopf fibration $\pi: S^{3} \rightarrow S^{2}$ we have the following corollary, which recovers [Inoguchi 2004, Proposition 3.1].

Corollary 4.1. There is no proper biharmonic Hopf cylinder in $S^{3}$.

Finally, applying (16) to the submersions $\pi: S^{2} \times \mathbb{R} \rightarrow S^{2}$ and $\pi: H^{2} \times \mathbb{R} \rightarrow H^{2}$ yields another corollary:

Corollary 4.2. (1) The Hopf cylinder $\Sigma=\bigcup_{t \in I} \pi^{-1}(\alpha(t))$ is a proper biharmonic surface in $S^{2} \times \mathbb{R}$ if and only if the directrix $\alpha: I \rightarrow\left(S^{2}, h\right)$ is a part of a circle in $S^{2}$ with radius $\sqrt{2} / 2$;

(2) The Hopf cylinder $\Sigma=\bigcup_{t \in I} \pi^{-1}(\alpha(t))$ is biharmonic in $H^{2} \times \mathbb{R}$ if and only if it is minimal.

\section{Acknowledgment}

I would like to thank the referee for many invaluable comments and suggestions that helped to clarify and improve the original manuscript.

\section{References}

[Arslan et al. 2005] K. Arslan, R. Ezentas, C. Murathan, and T. Sasahara, "Biharmonic submanifolds in 3-dimensional ( $\kappa, \mu)$-manifolds", Int. J. Math. Math. Sci. 22 (2005), 3575-3586. MR 2006k: 53108 Zbl 1096.53031

[Baird and Wood 2003] P. Baird and J. C. Wood, Harmonic morphisms between Riemannian manifolds, London Mathematical Society Monographs (N.S.) 29, Oxford University Press, 2003. MR 2005b:53101 Zbl 1055.53049

[Balmuş et al. 2007] A. Balmuş, S. Montaldo, and C. Oniciuc, "Biharmonic maps between warped product manifolds", J. Geom. Phys. 57:2 (2007), 449-466. MR 2007j:53066 Zbl 1108.58011

[Balmuş et al. 2008] A. Balmuş, S. Montaldo, and C. Oniciuc, "Classification results for biharmonic submanifolds in spheres", Israel J. Math. 168 (2008), 201-220. MR 2009j:53079 Zbl 1172.58004

[Besse 2008] A. L. Besse, Einstein manifolds, Springer, Berlin, 2008. MR 2008k:53084 Zbl 1147. 53001

[Caddeo et al. 2001] R. Caddeo, S. Montaldo, and C. Oniciuc, "Biharmonic submanifolds of $S^{3}$ ", Internat. J. Math. 12:8 (2001), 867-876. MR 2002k:53123 Zbl 1111.53302

[Caddeo et al. 2002] R. Caddeo, S. Montaldo, and C. Oniciuc, "Biharmonic submanifolds in spheres", Israel J. Math. 130 (2002), 109-123. MR 2003c:53090 Zbl 1038.58011

[Chen 1991] B.-Y. Chen, "Some open problems and conjectures on submanifolds of finite type", Soochow J. Math. 17:2 (1991), 169-188. MR 92m:53091 Zbl 0749.53037 
[Chen and Ishikawa 1998] B.-Y. Chen and S. Ishikawa, "Biharmonic pseudo-Riemannian submanifolds in pseudo-Euclidean spaces", Kyushu J. Math. 52:1 (1998), 167-185. MR 99b:53078 Zbl 0892.53012

[Dimitrić 1992] I. Dimitrić, "Submanifolds of $E^{m}$ with harmonic mean curvature vector", Bull. Inst. Math. Acad. Sinica 20:1 (1992), 53-65. MR 93g:53087 Zbl 0778.53046

[Fetcu and Oniciuc 2009a] D. Fetcu and C. Oniciuc, "Explicit formulas for biharmonic submanifolds in Sasakian space forms", Pacific J. Math. 240:1 (2009), 85-107. MR 2010c:53092 Zbl 1167.53024

[Fetcu and Oniciuc 2009b] D. Fetcu and C. Oniciuc, "On the geometry of biharmonic submanifolds in Sasakian space forms", J. Geom. Symmetry Phys. 14 (2009), 21-34. MR 2536497 Zbl 05580885

[Hasanis and Vlachos 1995] T. Hasanis and T. Vlachos, "Hypersurfaces in $E^{4}$ with harmonic mean curvature vector field”, Math. Nachr. 172 (1995), 145-169. MR 96c:53085 Zbl 0839.53007

[Inoguchi 2004] J.-I. Inoguchi, "Submanifolds with harmonic mean curvature vector field in contact 3-manifolds", Colloq. Math. 100:2 (2004), 163-179. MR 2005h:53105 Zbl 1076.53065

[Javaloyes and Meroño 2003] M. A. Javaloyes Victoria and M. A. Meroño Bayo, "Biharmonic lifts by means of pseudo-Riemannian submersions in dimension three", Trans. Amer. Math. Soc. 355:1 (2003), 169-176. MR 2003f:53107 Zbl 1020.53033

[Jiang 1986a] G. Y. Jiang, "2-harmonic isometric immersions between Riemannian manifolds", Chinese Ann. Math. Ser. A 7:2 (1986), 130-144. In Chinese; English summary in Chinese Ann. Math. Ser. B 7:2 (1986), 255. MR 87k:53140 Zbl 0596.53046

[Jiang 1986b] G. Y. Jiang, "2-harmonic maps and their first and second variational formulas", Chinese Ann. Math. Ser. A 7:4 (1986), 389-402. In Chinese; English summary in Chinese Ann. Math. Ser. B 7:4 (1986), 523. MR 88i:58039 Zbl 0628.58008

[Jiang 1987] G. Y. Jiang, "Some nonexistence theorems on 2-harmonic and isometric immersions in Euclidean space", Chinese Ann. Math. Ser. A 8:3 (1987), 377-383. In Chinese; English summary in Chinese Ann. Math. Ser. B 8:3 (1987), 389. MR 89a:53071 Zbl 0637.53071

[Kobayashi and Nomizu 1969] S. Kobayashi and K. Nomizu, Foundations of differential geometry, vol. 2, Pure and Applied Mathematics 15, Wiley, New York, 1969. MR MR0238225 (38 \#6501) Zbl 0175.48504

[Montaldo and Oniciuc 2006] S. Montaldo and C. Oniciuc, "A short survey on biharmonic maps between Riemannian manifolds", Rev. Un. Mat. Argentina 47:2 (2006), 1-22. MR 2008a:53063 Zbl 1140.58004

[Nishikawa and Maeda 1974] S. Nishikawa and Y. Maeda, "Conformally flat hypersurfaces in a conformally flat Riemannian manifold”, Tôhoku Math. J. (2) 26 (1974), 159-168. MR 49 \#3730 Zbl 0278.53018

[O’Neill 1966] B. O’Neill, “The fundamental equations of a submersion”, Michigan Math. J. 13 (1966), 459-469. MR 34 \#751 Zbl 0145.18602

[Oniciuc 2002] C. Oniciuc, "Biharmonic maps between Riemannian manifolds", An. Ştiint. Univ. Al. I. Cuza Iaşi. Mat. (N.S.) 48:2 (2002), 237-248 (2003). MR 2004e:53097 Zbl 1061.58015

[Ou 2009] Y.-L. Ou, "On conformal biharmonic immersions", Ann. Global Anal. Geom. 36:2 (2009), 133-142. MR 2010g:53118 Zbl 1178.53054

[Sasahara 2005] T. Sasahara, "Legendre surfaces in Sasakian space forms whose mean curvature vectors are eigenvectors", Publ. Math. Debrecen 67:3-4 (2005), 285-303. MR 2006c:53064

[Sasahara 2008] T. Sasahara, "Stability of biharmonic Legendrian submanifolds in Sasakian space forms”, Canad. Math. Bull. 51:3 (2008), 448-459. MR 2009c:53078 Zbl 1147.53315 
[Zhang 2008] W. Zhang, "New examples of biharmonic submanifolds in $\mathbb{C} P^{n}$ and $S^{2 n+1}$ ", preprint, 2008. arXiv 0705.3961

Received July 23, 2009. Revised October 31, 2009.

Ye-LIN OU

Department of Mathematics

TEXAS A\&M UNIVERSITY-COMMERCE

COMMERCE TX, 75429

UNITED STATES

yelin_ou@tamu-commerce.edu

www.tamu-commerce.edu 


\title{
PACIFIC JOURNAL OF MATHEMATICS
}

\author{
http://www.pjmath.org \\ Founded in 1951 by \\ E. F. Beckenbach (1906-1982) and F. Wolf (1904-1989)
}

\section{EDITORS}

V. S. Varadarajan (Managing Editor)

Department of Mathematics

University of California

Los Angeles, CA 90095-1555

pacific@math.ucla.edu

Vyjayanthi Chari

Department of Mathematics

University of California

Riverside, CA 92521-0135

chari@math.ucr.edu

Robert Finn

Department of Mathematics Stanford University

Stanford, CA 94305-2125

finn@math.stanford.edu

Kefeng Liu

Department of Mathematics

University of California

Los Angeles, CA 90095-1555

liu@math.ucla.edu
Darren Long

Department of Mathematics

University of California

Santa Barbara, CA 93106-3080

long@math.ucsb.edu

Jiang-Hua Lu

Department of Mathematics

The University of Hong Kong

Pokfulam Rd., Hong Kong jhlu@maths.hku.hk

Alexander Merkurjev

Department of Mathematics

University of California

Los Angeles, CA 90095-1555

merkurev@math.ucla.edu
Sorin Popa

Department of Mathematics University of California

Los Angeles, CA 90095-1555 popa@math.ucla.edu

Jie Qing

Department of Mathematics

University of California

Santa Cruz, CA 95064

qing@cats.ucsc.edu

Jonathan Rogawski

Department of Mathematics

University of California

Los Angeles, CA 90095-1555

jonr@math.ucla.edu

\section{PRODUCTION}

pacific@math.berkeley.edu

\begin{abstract}
Silvio Levy, Scientific Editor Matthew Cargo, Senior Production Editor
\end{abstract}
ACADEMIA SINICA, TAIPEI

CALIFORNIA INST. OF TECHNOLOGY

INST. DE MATEMÁTICA PURA E APLICADA

KEIO UNIVERSITY

MATH. SCIENCES RESEARCH INSTITUTE

NEW MEXICO STATE UNIV.

OREGON STATE UNIV.

\section{SUPPORTING INSTITUTIONS}

STANFORD UNIVERSITY
UNIV. OF BRITISH COLUMBIA
UNIV. OF CALIFORNIA, BERKELEY
UNIV. OF CALIFORNIA, DAVIS
UNIV. OF CALIFORNIA, LOS ANGELES
UNIV. OF CALIFORNIA, RIVERSIDE
UNIV. OF CALIFORNIA, SAN DIEGO
UNIV. OF CALIF., SANTA BARBARA

UNIV. OF CALIF., SANTA CRUZ

UNIV. OF MONTANA

UNIV. OF OREGON

UNIV. OF SOUTHERN CALIFORNIA

UNIV. OF UTAH

UNIV. OF WASHINGTON

WASHINGTON STATE UNIVERSITY

These supporting institutions contribute to the cost of publication of this Journal, but they are not owners or publishers and have no responsibility for its contents or policies.

See inside back cover or www.pjmath.org for submission instructions.

The subscription price for 2010 is US \$420/year for the electronic version, and \$485/year for print and electronic.

Subscriptions, requests for back issues from the last three years and changes of subscribers address should be sent to Pacific Journal of Mathematics, P.O. Box 4163, Berkeley, CA 94704-0163, U.S.A. Prior back issues are obtainable from Periodicals Service Company, 11 Main Street, Germantown, NY 12526-5635. The Pacific Journal of Mathematics is indexed by Mathematical Reviews, Zentralblatt MATH, PASCAL CNRS Index, Referativnyi Zhurnal, Current Mathematical Publications and the Science Citation Index.

The Pacific Journal of Mathematics (ISSN 0030-8730) at the University of California, c/o Department of Mathematics, 969 Evans Hall, Berkeley, CA 94720-3840, is published monthly except July and August. Periodical rate postage paid at Berkeley, CA 94704, and additional mailing offices. POSTMASTER: send address changes to Pacific Journal of Mathematics, P.O. Box 4163, Berkeley, CA 94704-0163.

PJM peer review and production are managed by EditFLOW ${ }^{\mathrm{TM}}$ from Mathematical Sciences Publishers.

PUBLISHED BY PACIFIC JOURNAL OF MATHEMATICS

at the University of California, Berkeley 94720-3840

A NON-PROFIT CORPORATION

Typeset in LATEX

Copyright $(\mathrm{C} 2010$ by Pacific Journal of Mathematics 


\section{PACIFIC JOURNAL OF MATHEMATICS}

Volume $248 \quad$ No. $1 \quad$ November 2010

An existence theorem of conformal scalar-flat metrics on manifolds with boundary 1

SÉRgio de Moura Almaraz

Parasurface groups

KHALID BOU-RABEE

Expressions for Catalan Kronecker products

Andrew A. H. Brown, Stephanie Van Willigenburg and Mike

ZABROCKI

Metric properties of higher-dimensional Thompson's groups

José BURILLO and SEAN CLEARY

Solitary waves for the Hartree equation with a slowly varying potential

KIRIL DATCHEV and IVAN VENTURA

Uniquely presented finitely generated commutative monoids

PEDRO A. GARCíA-SÁNCHEZ and IgnaCio OJEDA

The unitary dual of $p$-adic $\widetilde{\operatorname{Sp}(2)}$

MARCELA HANZER and IVAN MATIĆ

A Casson-Lin type invariant for links

ERIC HARPER and NIKOLAI SAVELIEV

Semiquandles and flat virtual knots

ALLISON HENRICH and SAM NELSON

Infinitesimal rigidity of polyhedra with vertices in convex position

IVAN IZMESTIEV and JEAN-MARC SCHLENKER

Robust four-manifolds and robust embeddings

VYACHESLAV S. KRUSHKAL

On sections of genus two Lefschetz fibrations

SINEM ÇELIK ONARAN

Biharmonic hypersurfaces in Riemannian manifolds

YE-LIN OU

Singular fibers and 4-dimensional cobordism group 